\title{
Performance of Multihop Wireless Links over Generalized- $K$ Fading Channels
}

\author{
Jianfei Cao ${ }^{1}$, Lie-Liang Yang ${ }^{2}$ and Zhangdui Zhong ${ }^{1}$ \\ ${ }^{1}$ State Key Lab of Rail Traffic Control and Safety \\ Beijing Jiaotong University, Beijing 100044, China \\ Emails: jc09v@ecs.soton.ac.uk, zhdzhong@bjtu.edu.cn \\ ${ }^{2}$ School of ECS, University of Southampton \\ SO17 1BJ, United Kingdom, Email: 1ly@ecs.soton.ac.uk
}

\begin{abstract}
The performance of multihop links is studied in this contribution by both analysis and simulations, when communicating over Generalized- $K\left(K_{G}\right)$ fading channels. The performance metrics considered include symbol error rate (SER), outage probability, level crossing rate $(\mathrm{LCR})$ and average outage duration (AOD). First, the expressions for both the SER and outage probability are derived by approximating the probability density function (PDF) of the end-to-end signal-to-noise ratio (SNR) using an equivalent end-to-end PDF. We show that this equivalent endto-end PDF is accurate for analyzing the outage probability. Then, the second-order statistics of LCR and AOD of multihop links are analyzed. Finally, the performance of multihop links is investigated either by simulations or by evaluation of the expressions derived. Our performance results show that the analytical expressions obtained can be well justified by the simulation results. The studies show that the $K_{G}$ channel model as well as the expressions derived in this paper are highly efficient for predicting the various types of performance metrics and statistics for design of multihop communication links.
\end{abstract}

\section{INTRODUCTION}

In mobile wireless communications systems, radio signals experience propagation path-loss, shadowing and multipath introduced fast fading [1]. Conventionally, the effect of wireless channels on system performance is investigated by treating these phenomena separately, assuming typically 2-4 order of power decay for the propagation path-loss, lognormal distribution for shadowing and Rayleigh, Rician or Nakagami- $m$ distribution for fast fading [2]. In order to predict accurately the achievable performance of a communication scheme, it is usually desirable that a model can simultaneously cope with all the above-mentioned three phenomena. For this sake, composite channel models have been proposed, which are often formed by combining distributions for fast fading with lognormal distribution for shadowing, yielding Rayleigh-, Rician, Nakagami-lognormal, etc., composite channel models [2]. However, these composite channel models usually result in complicated performance analysis.

In recent years, the generalized- $K\left(K_{G}\right)$ channel model has been proposed [3], which is formed by, first, approximating the lognormal distribution using the Gamma distribution [2] and, then, combining it with the Nakagami- $m$ distribution. It has been recognized [3] that the $K_{G}$ channel model can simultaneously take into account of the propagation path-loss, shadowing and fast fading. It can usually covers more communications scenarios encountered in real mobile wireless systems, than the other composite channel models. Furthermore, the $K_{G}$ channel model often results in closed-form solutions when applied for system performance analysis [4-6]. Therefore, in this paper we motivate to analyze the performance of multihop links, when they are operated in $K_{G}$ fading channels. The symbol error rate (SER), outage probability, level crossing rate (LCR) and average outage duration (AOD) of the multihop links are analyzed.

By dividing a long transmission link into multiple more reliable short links supported by relays, multihop transmission has the potential to improve the system's power-efficiency and extend the coverage area. Recently, the performance analysis of relay networks has drawn a lot of attention, as evidenced, e.g., by the references [7-10] as well as their related references. However, to the best of our knowledge, the performance, especially, the second order statistics of LCR and AOD, of multihop links with regenerative relays for communications over $K_{G}$ fading channels has not been analyzed in open literature. In this paper, a range of closed-form expressions for the SER, outage probability, LCR and AOD of multihop links are derived, when the multihop links are represented by their corresponding equivalent point-to-point links. Finally, the performance of the multihop links is investigated by simulations and/or by evaluation of the formulas derived.

\section{Statistics OF END-To-End SNR}

\section{A. System and Channel Model}

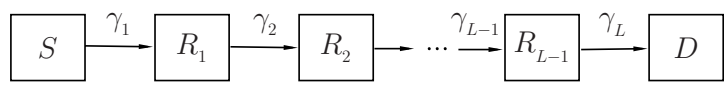

Fig. 1. Schematic of a multihop transmission link, where source $S$ sends messages to destination $D$ through $(L-1)$ relays, the SNR of the $l$ th hop is denoted by $\gamma_{l}$ for $l=1,2, \ldots, L$.

Consider a $L$-hop link as depicted in Fig. 1, where the source $S$ sends messages to the destination $D$ via $(L-1)$ intermediate relays, expressed as $R_{1}, R_{2}, \ldots, R_{L-1}$, respectively. The relays are operated under the decode-and-forward (DF) cooperative strategy. The SNRs of the $L$ hops are denoted by $\gamma_{1}, \gamma_{2}, \ldots, \gamma_{L}$, respectively. This type of communication links exist in wireless ad-hoc networks, wireless sensor networks, etc.

We assume that the fading envelopes $Z_{1}, Z_{2}, \ldots, Z_{L}$ of the 
$L$ hops obey the $K_{G}$ distribution with the PDF [4]

$$
\begin{aligned}
f_{Z_{l}}\left(z_{l}\right)= & \frac{4 z_{l}^{\beta_{l}}}{\Gamma\left(m_{l}\right) \Gamma\left(k_{l}\right)}\left(\frac{m_{l} k_{l}}{\Omega_{l}}\right)^{\frac{\beta_{l}+1}{2}} K_{\alpha_{l}}\left[2 \sqrt{\frac{m_{l} k_{l}}{\Omega_{l}}} z_{l}\right], \\
& z_{l} \geq 0, l=1,2, \ldots, L
\end{aligned}
$$

where $\alpha_{l}=k_{l}-m_{l}$ and $\beta_{l}=k_{l}+m_{l}-1, k_{l}$ and $m_{l}$ are the distribution shaping parameters accounting for the shadowing and Nakagami- $m$ fading associated with the $l$ th hop, $K_{\alpha_{l}}(\cdot)$ is the second kind modified Bessel function of order $\alpha_{l}$, while $\Gamma(\cdot)$ denotes the Gamma function. In (1), $\Omega_{l}=E\left\{Z_{l}^{2}\right\}$, where $E\{\cdot\}$ is the expectation operation, is the mean of the shadowing fading, which is determined by propagation loss. Therefore, by controlling the values of the shaping parameters $k_{l}$ and $m_{l}$ as well as the value of $\Omega_{l}$, (1) can be used to approximate the signal distribution in numerous communication environments. Multipath fading, shadowing, the composite of shadowing and multipath fading, additive white Gaussian noise (AWGN) channels, etc., can all be modelled by the distribution of (1) by setting appropriately the parameters of $k_{l}, m_{l}$ and $\Omega_{l}$ [6].

Let $\gamma_{l}=Z_{l}^{2} E / N_{0}$ denote the instantaneous SNR of the $l$ th hop, where $E$ denotes the energy per symbol and $N_{0}$ the singlesided power spectral density (PSD) of AWGN. The PDF of $\gamma_{l}$ can be readily derived from (1), which is

$$
f_{\gamma_{l}}(\gamma)=\frac{2 \Psi_{l}^{\left(\beta_{l}+1\right) / 2} \gamma^{\left(\beta_{l}-1\right) / 2}}{\Gamma\left(m_{l}\right) \Gamma\left(k_{l}\right)} K_{\alpha_{l}}\left[2 \sqrt{\Psi_{l} \gamma}\right], \gamma \geq 0
$$

where $\Psi_{l}=m_{l} k_{l} / \bar{\gamma}_{l}$ and $\bar{\gamma}_{l}=\Omega_{l} E / N_{0}$ is the average SNR of the $l$ th hop.

According to [11], the cascaded multihop link from source $S$ to destination $D$ in Fig. 1 can be reduced to an equivalent pointto-point link, in order to simplify the analysis. With the aid of this equivalent model, the PDF of the end-to-end SNR of Fig. 1 can be approximated by [11]

$$
f(\gamma)=A \delta(\gamma)+(1-A) f_{\gamma_{L}}(\gamma)
$$

where $A$ is the probability that outage occurs with the first $(L-1)$ hops, while $f_{\gamma_{L}}(\gamma)$ denotes the PDF of the last hop's SNR. Explicitly, the equivalent PDF of the end-to-end SNR is constituted by two components: the probability of $P(\gamma=0)=$ $A$ and the $K_{G}$ PDF scaled by a factor $(1-A)$. We assume that the SNRs, $\gamma_{l}, l=1, \ldots, L$, are mutually independent and have the PDFs $f_{\gamma_{l}}(\gamma), l=1, \ldots, L$, that may be different. Then, the probability $A$ in (3) is

$$
\begin{aligned}
A & =1-\operatorname{Pr}\left\{\gamma_{1}>\gamma_{t h}, \gamma_{2}>\gamma_{t h}, \ldots, \gamma_{L-1}>\gamma_{t h}\right\} \\
& =1-\prod_{l=1}^{L-1}\left[1-F_{\gamma_{l}}\left(\gamma_{t h}\right)\right]
\end{aligned}
$$

where $F_{\gamma_{l}}(\cdot)$ represents the cumulative distribution function (CDF) of $\gamma_{l}$, which, for non-integer parameter $\alpha_{l}$, is given by [6]

$$
\begin{gathered}
F_{\gamma_{l}}(\gamma)=\pi \csc \left(\pi \alpha_{l}\right)\left[\frac{\left(\Psi_{l} \gamma\right)^{m_{l}}{ }_{1} F_{2}\left(m_{l} ; 1-\alpha_{l}, 1+m_{l} ; \Psi_{l} \gamma\right)}{\Gamma\left(k_{l}\right) \Gamma\left(1-\alpha_{l}\right) \Gamma\left(1+m_{l}\right)}\right. \\
\left.-\frac{\left(\Psi_{l} \gamma\right)^{k_{l}}{ }_{1} F_{2}\left(k_{l} ; 1+\alpha_{l}, 1+k_{l} ; \Psi_{l} \gamma\right)}{\Gamma\left(m_{l}\right) \Gamma\left(1+\alpha_{l}\right) \Gamma\left(1+k_{l}\right)}\right], \gamma \geq 0
\end{gathered}
$$

where ${ }_{p} F_{q}$ is the generalized hyper-geometric function with integer parameters $p$ and $q$ [12]. When the parameters $m_{l}$ and $k_{l}$ are integers, then, the CDF of $\gamma_{l}$ can be expressed as [13]

$$
\begin{gathered}
F_{\gamma_{l}}(\gamma)=1-\frac{2\left(\Psi_{l} \gamma\right)^{k_{l} / 2}}{\Gamma\left(k_{l}\right)} \sum_{q=0}^{m_{l}-1} \frac{1}{q !}\left(\Psi_{l} \gamma\right)^{q / 2} K_{k_{l}-q}\left[2 \sqrt{\Psi_{l} \gamma}\right] \\
\gamma>0 .
\end{gathered}
$$

\section{B. Amount of Fading}

With the equivalent PDF as shown in (3), the $n$th moment of the end-to-end SNR $\mu_{\gamma}(n)$ can be evaluated by the formula

$$
\mu_{\gamma}(n)=\int_{0}^{\infty} \gamma^{n} f(\gamma) d \gamma
$$

Substituting (3) into (7) and using [12, eq. (6.561/16)] gives

$$
\mu_{\gamma}(n)=(1-A) \frac{\Gamma\left(k_{L}+n\right) \Gamma\left(m_{L}+n\right)}{\Gamma\left(k_{L}\right) \Gamma\left(m_{L}\right) \Psi_{L}^{n}} .
$$

The amount of fading (AoF) used to measure the severity of fading can then be computed by the formula [2]

$$
A o F=\frac{\mu_{\gamma}(2)}{\mu_{\gamma}^{2}(1)}-1 .
$$

\section{Analysis of SER AND OUtage Probability}

The moment generation function (MGF) [2] is adopted for deriving the expressions for the SER and outage probabilities. The MGF, $M_{\gamma}(s)$, of the end-to-end SNR can be expressed as

$$
M_{\gamma}(s)=\int_{0}^{\infty} \exp (-\gamma s) f(\gamma) d \gamma .
$$

By substituting (3) into (10) and using [12, eq. (6.643/3)], we can obtain

$$
\begin{aligned}
M_{\gamma}(s)=A+(1-A) & \left(\frac{\Psi_{L}}{s}\right)^{\frac{\beta_{L}}{2}} \exp \left(\frac{\Psi_{L}}{2 s}\right) \\
& \times W_{-\frac{\beta_{L}}{2}, \frac{\alpha_{L}}{2}}\left(\frac{\Psi_{L}}{s}\right),
\end{aligned}
$$

where $W_{v, \mu}(z)$ is the Whittaker's function [12].

\section{A. Average Symbol Error Rate}

For many coherent demodulation schemes, such as $M$ ary amplitude shift-keying (MASK), binary phase shift-keying (BPSK), binary frequency shift-keying (BFSK), etc., the SER $P_{e}(\gamma)$ conditioned on the SNR $\gamma$ can be expressed as $[2,14]$

$$
P_{e}(\gamma)=a \int_{0}^{\frac{\pi}{2}} \exp \left(-\frac{g \gamma}{\sin ^{2} \theta}\right) d \theta
$$

where the parameters $a$ and $g$ are specified by the modulation scheme employed $[2,14]$. The average SER can be evaluated by integrating $P_{e}(\gamma)$ with respect to the PDF of $\gamma$, which it can be shown yields

$$
\bar{P}_{e}=a \int_{0}^{\frac{\pi}{2}} M_{\gamma}\left(\frac{g}{\sin ^{2} \theta}\right) d \theta,
$$


Upon applying (11) into (13) and completing some simplification, we finally obtain the average SER

$$
\begin{aligned}
& \bar{P}_{e}=\frac{\pi a A}{2}+\frac{a(1-A) \Gamma\left(\frac{1}{2}\right)}{2 \Gamma\left(\frac{1-\alpha_{L}+\beta_{L}}{2}\right) \Gamma\left(\frac{1+\alpha_{L}+\beta_{L}}{2}\right)}\left(\frac{\Psi_{L}}{g}\right)^{\frac{\beta_{L}}{2}} \\
& \times G_{2,3}^{2,2}\left(\begin{array}{c|c}
\Psi_{L} & \left(1-\beta_{l}\right) / 2,\left(2-\beta_{l}\right) / 2 \\
g & \left(1+\alpha_{l}\right) / 2,\left(1-\alpha_{l}\right) / 2,-\beta_{l} / 2
\end{array}\right),
\end{aligned}
$$

where $G(\cdot)$ is the Meijer's $G$-function defined by [12, eq. (9.301)].

When non-coherent demodulation, such as BFSK, etc., employing square-law detection is considered, the conditional SER can be expressed as $P_{e, n o n}(\gamma)=C \exp (-D \gamma)$, where $C$ and $D$ are constants specified by the corresponding noncoherent modulation scheme. By averaging $P_{e, n o n}(\gamma)$ using the PDF of (3) and with the aid of [12, eq. 6.643/3], we can obtain the average SER

$$
\begin{aligned}
\bar{P}_{e, n o n}= & C A+C(1-A)\left(\frac{\Psi_{L}}{D}\right)^{\frac{\beta_{L}}{2}} \\
& \times \exp \left(\frac{\Psi_{L}}{2 D}\right) W_{-\frac{\beta_{L}}{2}, \frac{\alpha_{L}}{2}}\left(\frac{\Psi_{L}}{D}\right) .
\end{aligned}
$$

\section{B. Outage Probability}

The outage event occurs provided that there is at least a hop having its SNR lower than a threshold $\gamma_{t h}$, i.e., $P_{\text {out }}=$ $\int_{0}^{\gamma_{t h}} f(\gamma) d \gamma$. Substituting (3) into this equation gives

$$
P_{\text {out }}=A+(1-A) F_{\gamma_{L}}\left(\gamma_{t h}\right)
$$

The outage probability can also be directly derived from the multihop links of Fig. 1, which is

$$
\begin{aligned}
P_{\text {out }} & =1-P\left[\gamma_{1}>\gamma_{t h}, \gamma_{2}>\gamma_{t h}, \ldots, \gamma_{L}>\gamma_{t h}\right] \\
& =1-\prod_{l=1}^{L}\left[1-F_{\gamma_{l}}\left(\gamma_{t h}\right)\right] .
\end{aligned}
$$

Let in this equation $\bar{A}=\prod_{l=1}^{L-1}\left[1-F_{\gamma_{l}}\left(\gamma_{t h}\right)\right]$. According to (4), explicitly, we have $A+\bar{A}=1$. Upon applying them into (17), we can readily proof that (17) is the same as (16). This implies that the equivalent model associated with the PDF of (3) is accurate in terms of the outage probability.

\section{Level Crossing Rate And Average Outage DURATION}

In this section, we first derive the LCR and AOD in the context of the $l$ th hop. Then, the results are extended to the multihop paradigms with the aid of the approaches proposed in [9]. For simplicity, the subscript $l$ is first dropped, when without any confusion.

According to [15], the AOD, $T\left(z_{t h}\right)$, in seconds can be approximately expressed as

$$
T\left(z_{t h}\right) \approx P_{\text {out }} / N\left(z_{t h}\right)
$$

where $P_{\text {out }}$ is the outage probability having been derived in Section III-B and $N\left(z_{t h}\right)$ is the LCR at a given threshold $z_{t h}$. According to [16], the LCR $N\left(z_{t h}\right)$ can be evaluated by the integration

$$
N\left(z_{t h}\right)=\int_{0}^{\infty} \dot{z} f_{Z \dot{Z}}\left(z_{t h}, \dot{z}\right) d \dot{z}
$$

where $f_{Z \dot{z}}(z, \dot{z})$ represents the joint PDF of the random process $Z(t)$ and its time derivative $\dot{Z}=d Z(t) / d t$, both evaluated at a fixed time $t$. Specifically, for a random process $Z(t)$ with its samples obeying the $K_{G}$ distribution, we find after some highly involved analysis that the joint PDF $f_{Z \dot{Z}}(z, \dot{z})$ can be expressed as

$$
\begin{array}{r}
f_{Z, \dot{Z}}(z, \dot{z})=\frac{2 \sqrt{a b} m^{m} k^{k} z^{2 m-1}}{\sqrt{\pi} \Gamma(m) \Gamma(k) \Omega^{k}} \int_{0}^{\infty} \frac{y^{\alpha}}{\sqrt{a y^{3}+b z^{2} y}} \\
\quad \times \exp \left(-\frac{k}{\Omega} y-\frac{b \dot{z}+m z^{2}}{y}+\frac{b^{2} z^{2} z^{2}}{a y^{3}+b z^{2} y}\right) d y
\end{array}
$$

where, by definition, $a=-k / 4 \Omega \rho^{\prime \prime}(0)$ and $b=m / 2 \pi^{2} f_{m}^{2}$. The function $\rho(\tau)$ is the normalized correlation function of the Gamma distributed random process used for forming the $K_{G}$ distributed random process, and $\rho^{\prime \prime}(0)$ is its second order derivative at $\tau=0$. For example, for land mobile communication systems, usually $\rho(\tau)=J_{0}\left(2 \pi f_{m} \tau\right)$, where $J_{0}(x)$ is the zero-th order Bessel function of the first kind and $f_{m}$ denotes the maximum Doppler frequency-shift. In this case, we have $\rho^{\prime \prime}(0)=-2 \pi^{2} f_{m}^{2}$.

Let in (20) $z=z_{t h}$. Then, when substituting (20) into (19) and completing the integration with respect to $\dot{z}$, we can finally obtain the LCR for a single-hop link as

$$
\begin{aligned}
N\left(z_{t h}\right)= & \frac{m^{m} k^{k} z_{t h}^{2 m-1}}{\sqrt{a b \pi} \Gamma(m) \Gamma(k) \Omega^{k}} \int_{0}^{\infty} y^{\alpha-\frac{3}{2}} \sqrt{a y^{2}+b z_{t h}^{2}} \\
& \times \exp \left(-\frac{k}{\Omega} y-\frac{m z_{t h}^{2}}{y}\right) d y .
\end{aligned}
$$

When the multihop links are considered, according to [9], the end-to-end LCR over the $K_{G}$ channels can be evaluated by the formula

$$
N_{T}\left(z_{t h}\right)=\sum_{n=1}^{L} N_{n}\left(z_{t h}\right) \prod_{\substack{l=1 \\ l \neq n}}^{L}\left[1-F_{Z_{l}}\left(z_{t h}\right)\right]
$$

where $N_{n}(\cdot)$ denotes the $n$th hop's LCR, which is given by (21) with the parameters $m, k$, and $\Omega$ replaced by $m_{n}, k_{n}$ and $\Omega_{n}$, respectively. In (22), $F_{Z_{l}}(\cdot)$ denotes the CDF of $Z_{l}$ of the $l$ th hop's envelope, which can be derived from (5) and (6) based on the variable transform of $\gamma_{l}=\bar{\gamma}_{l} Z_{l}^{2} / \Omega_{l}$. From this variable transform, it can be shown that $F_{Z_{l}}(z)=F_{\gamma_{l}}\left(\bar{\gamma}_{l} z^{2} / \Omega_{l}\right)$. Hence, for non-integer parameter $\alpha_{l}$, we obtain from (5) that

$$
\begin{aligned}
& F_{Z_{l}}(z)=\pi \csc \left(\pi \alpha_{l}\right)\left[\left(\frac{m_{l} k_{l}}{\Omega_{l}} z^{2}\right)^{m_{l}}\right. \\
& \times \frac{{ }_{1} F_{2}\left(m_{l} ; 1-\alpha_{l}, 1+m_{l} ; m_{l} k_{l} z^{2} / \Omega_{l}\right)}{\Gamma\left(k_{l}\right) \Gamma\left(1-\alpha_{l}\right) \Gamma\left(1+m_{l}\right)}-\left(\frac{m_{l} k_{l}}{\Omega_{l}} z^{2}\right)^{k_{l}} \\
& \left.\quad \times \frac{{ }_{1} F_{2}\left(k_{l} ; 1+\alpha_{l}, 1+k_{l} ; m_{l} k_{l} z^{2} / \Omega_{l}\right)}{\Gamma\left(m_{l}\right) \Gamma\left(1+\alpha_{l}\right) \Gamma\left(1+k_{l}\right)}\right]
\end{aligned}
$$

By contrast, when $m_{l}$ and $k_{l}$ are integers, we can have from (6) that

$$
\begin{aligned}
F_{Z_{l}}(z)= & -\frac{2}{\Gamma\left(k_{l}\right)}\left(\frac{m_{l} k_{l}}{\Omega_{l}} z^{2}\right)^{k_{l} / 2} \sum_{q=0}^{m_{l}-1} \frac{1}{q !} \\
& \times\left(\frac{m_{l} k_{l}}{\Omega_{l}} z^{2}\right)^{q / 2} K_{k_{l}-q}\left[2 \sqrt{\frac{m_{l} k_{l}}{\Omega_{l}}} z\right]
\end{aligned}
$$


Finally, the AOD of multihop links communicating over $K_{G}$ channels can be obtained by substituting $P_{\text {out }}$ of (16) and $N_{T}\left(z_{t h}\right)$ of (22) into (18).

\section{Performance Results}

In the section the SER, outage probability, AoF, LCR and AOD performance of the multihop links communicating over $K_{G}$ fading channels are investigated by both simulation and numerical approaches. In our investigations, we assumed that the channels of the $L$ hops were independent identical distributed (iid) $K_{G}$ fading channels. The impact of propagation path-loss, shadowing and Nakagami- $m$ fast fading on the achievable performance was all considered. For convenience, the intermediate relays were assumed to be equally located on a line connecting the source $S$ and the destination $D$, making all the $L$ hops the same distance. We assumed that the maximum distance from the source $S$ to the destination $D$ was $L_{\max }=5$. Hence, when given $L \leq L_{\max }$, the parameter $\Omega_{l}$ in (1), which accounts for the propagation path-loss, was $\Omega_{l}=\Omega=\left(L / L_{\max }\right)^{\eta}$, where $\eta$ represents the global propagation path-loss exponent, which was set to $\eta=3$ in our simulation and numerical examples. Furthermore, for the sake of fairness of comparison, the transmission power was assumed to be evenly allocated to the $L$ transmitters, making $\bar{\gamma}_{l}=\bar{\gamma}=\Omega \times \mathrm{SNR} / L$, where SNR is the total SNR without propagation path-loss, which was used for depicting the figures. Additionally, when concerning the outage probability, the threshold was set according to $\log _{2}\left(1+\gamma_{t h}\right) / L=R$ with the spectrum efficiency $R$ being set to $0.3 \mathrm{bits} / \mathrm{s} / \mathrm{Hz}$.

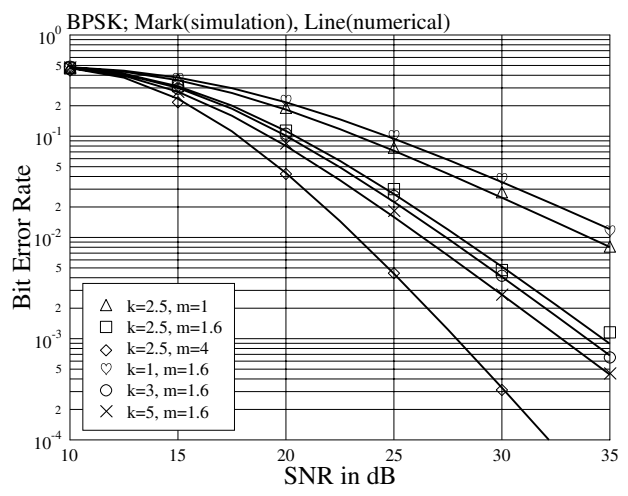

Fig. 2. BER performance of three-hop links using BPSK modulation, when communicating over $K_{G}$ fading channels with $\Omega_{l}=\Omega=(3 / 5)^{3}$ and other various shaping parameters.

Fig. 2 illustrates the average BER performance of the threehop $(L=3)$ links employing coherent BPSK modulation. From the results of Fig. 2, first, we can observe that the numerical results agree well with the simulation results. Therefore, the equivalent point-to-point link with the PDF given by (3) is an effective model for studying the error performance of a corresponding multihop link. Second, given a fixed value of $m=1.6$ corresponding to a fix fast fading, the BER performance of the multihop links improves, as the value of $k$ increases, implying that the channel becomes less shadowing. Similarly, for a given value of $k=2.5$, the BER performance improves, as the value of $m$ increases, corresponding to that the fast fading becomes less severe.

Figs. 3 and 4 investigate the effect of the number of hops on the achievable BER (Fig. 3) or outage (Fig. 4) performance.

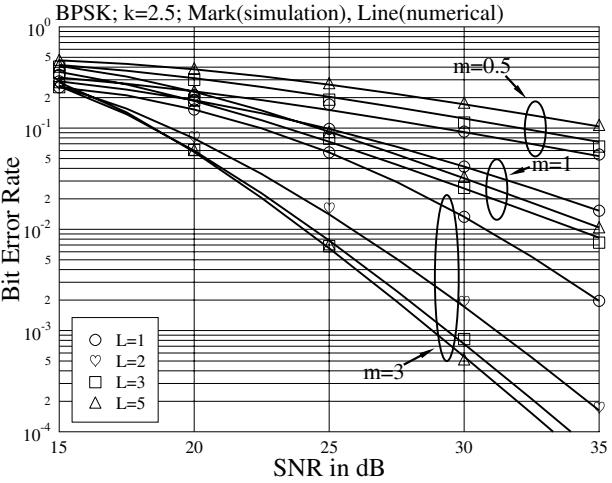

Fig. 3. BER performance of $L=1,2,3$ or 5 hop links using BPSK modulation, when communicating over $K_{G}$ fading channels with $\Omega_{l}=\Omega=$ $(L / 5)^{3}$.

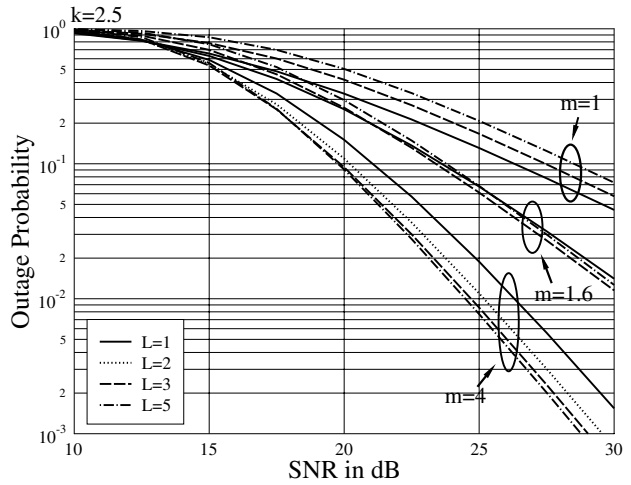

Fig. 4. Outage Probability of $L=1,2,3$ or 5 hop links, when communicating over $K_{G}$ fading channels with $\Omega_{l}=\Omega=(L / 5)^{3}$.

Explicitly, when given the total propagation distance $\left(L_{\max }=\right.$ 5 ), shadowing $k=2.5$ and the fast fading of a communication link, there exists an optimum number of hops, which yields the lowest BER or lowest outage probability. Specifically, when the fast fading is very severe corresponding to $m=0.5$ in Fig. 3 and $m=1$ in Fig. 4 using only one-hop link results in the best performance. When $m=1$ in Fig. 3 and $m=1.6$ in Fig. 4, depended on the available SNR, three-hop or five-hop link may achieve the best performance. Finally, when the channel quality in terms of the fast fading is very good, for example, when $m=$ 3 in Fig. 3 and $m=4$ in Fig. 4, generally, the link is beneficial to using as many hops as possible, in order to attain the best BER and outage performance.

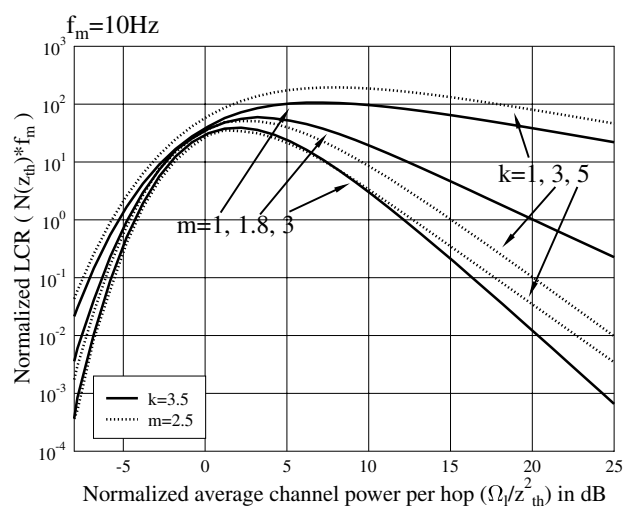

Fig. 5. Level-crossing rate of three-hop links, when communicating over $K_{G}$ fading channels with $\Omega_{l}=\Omega=(3 / 5)^{3}$ and other various shaping parameters as shown in the figure. 


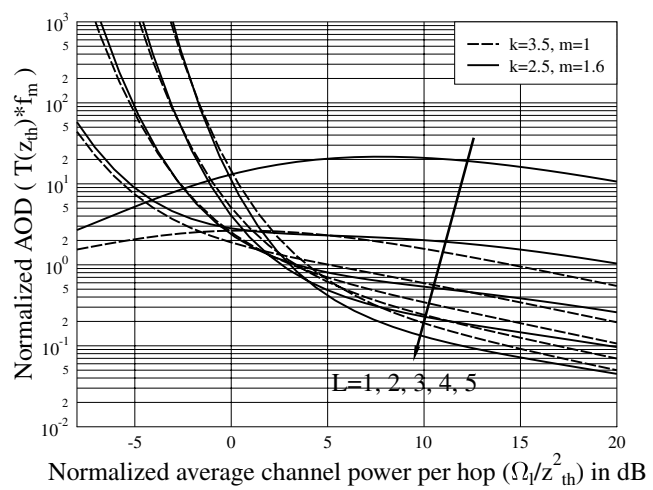

Fig. 6. Average outage duration of multihop links, when communicating over $K_{G}$ fading channels with $\Omega_{l}=\Omega=(3 / 5)^{3}$ and other various shaping parameters as shown in the figure.

In Fig. 5, we compare the end-to-end LCR of the fading over a three-hop link, when the communication channels over the three hops are modelled as the iid $K_{G}$ fading channels with various shaping parameters as shown in the figure. In Fig. 5, the results were numerically evaluated based on (22), when given the Doppler frequency-shift $f_{m}=10 \mathrm{~Hz}$. From the results of Fig. 5, it seems that the LCR of the three-hop link considered is sensitive more to the change of the fast fading determined by the parameter $m$ than to the varying of the shadowing explained by the parameter $k$. When the average channel power per hop is low, the LCR is low, since, in this case, the three-hop channel stays mainly at the 'poor' state without many chances going to the 'good' state. Similarly, when the average channel power is high, the LCR is also low. This is because, in this case, the three-hop channel stays mainly at the 'good' state without much fluctuation. By contrast, as shown in Fig. 5, there is a range for the average channel power, which resulting in that the three-hop channel is highly fluctuate, yielding relatively high LCR. Finally, from Fig. 5, we observe that, for a given value of average channel power, the three-hop channel becomes more fluctuate, as the value of $k$ or $m$ decreases.

Finally, Fig. 6 demonstrates the normalized AOD of the links having different number of hops. Note that, after normalized by the Doppler frequency-shift, the AOD is independent of $f_{m}$. As shown in Fig. 6, when the average channel power is low, such as $\Omega_{l} / z_{t h}^{2}<-5 \mathrm{~dB}$, the AOD of the one-hop link may be significantly shorter than the AOD of the multihop links. By contrast, when the average channel power is high, such as $\Omega_{l} / z_{t h}^{2}>5 \mathrm{~dB}$, the AOD decreases as the number of hops increases. The principles behind the above observation may be explained as follows. When the average channel power is low, each of the hops is an unreliable channel. Hence, the multihop link has a higher chance to stay at the 'poor' state, as the number of hops increase. By contrast, when the average channel power is high, due to the fluctuation of the component channels, the multihop channel becomes more fluctuate, as the number of hops increases. Correspondingly, the AOD of the multihop link becomes shorter, as the number of hops increases.

\section{CONCLUSION}

Our studies and performance results show that, the $K_{G}$ channel model as well as the expressions derived are highly efficient for evaluating the SER and outage performance multihop communication links, as well as for revealing the statistical behaviour of multihop communication links. Given the distance of a transmission link and the corresponding fading channel determined by the parameters for the propagation path-loss, shadowing and fast fading, our studies show that there exists an optimum number of hops for signal delivery, which results in the best SER and outage performance. In general, when the channel is very poor, one-hop direct transmission is the desired signal transmission option. When the quality of the channels improves, the communication link expects more hops in order to augment the SER and outage performance. If the channels are very reliable, it seems that the best SER and outage performance are attained, when the communication link uses as many hops as possible.

\section{ACKNOWLEDGEMENT}

The authors would like to acknowledge with thanks the financial assistance from the Joint State Key Program of National Natural Science Fundation of China (Grant No: 60830001).

\section{REFERENCES}

[1] T. S. Rappaport, Wireless Communications Principles and Practice, 2nd ed. New York: Prentice Hall, Inc, 2002.

[2] M. K. Simon and M.-S. Alouini, Digital Communication over Fading Channels. New York: Wiley, 2005.

[3] A. Abdi and M. Kaveh, "On the utility of gamma pdf in modeling shadow fading (slow fading)," Vehicular Technology Conference, 1999 IEEE 49th, vol. 3, pp. $2308-2312$ vol.3, Jul 1999.

[4] P. M. Shankar, "Error rates in generalized shadowed fading channels," Wirel. Pers. Commun., vol. 28, no. 3, pp. 233-238, 2004.

[5] P. Shankar, "Outage probabilities in shadowed fading channels using a compound statistical model," Communications, IEE Proceedings-, vol. 152, no. 6, pp. 828-832, Dec. 2005.

[6] P. Bithas, N. Sagias, P. Mathiopoulos, G. Karagiannidis, and A. Rontogiannis, "On the performance analysis of digital communications over generalized-k fading channels," Communications Letters, IEEE, vol. 10, no. 5, pp. 353-355, May 2006.

[7] M. Hasna and M.-S. Alouini, "Outage probability of multihop transmission over nakagami fading channels," Communications Letters, IEEE vol. 7, no. 5, pp. 216-218, May 2003.

[8] L. Wu, J. Lin, K. Niu, and Z. He, "Performance of dual-hop transmissions with fixed gain relays over generalized-k fading channels," in Communications, 2009. ICC '09. IEEE International Conference on, June 2009, pp. 1-5.

[9] L. Yang, M. Hasna, and M.-S. Alouini, "Average outage duration of multihop communication systems with regenerative relays," Wireless Communications, IEEE Transactions on, vol. 4, no. 4, pp. 1366-1371, July 2005.

[10] A. Krantzik and D. Wolf, "Distribution of the fading-intervals of modified suzuki processes," Signal Processing V: Theories and Applications, L.Torres, E. Masgrau, and M. A. Lagunas, Eds. Amsterdam, The Netherlands: Elsevier Science, pp. 361-364, 1990.

[11] N. C. Beaulieu and J. Hu, "A closed-form expression for the outage probability of decode-and-forward relaying in dissimilar rayleigh fading channels," Communications Letters, IEEE, vol. 10, no. 12, pp. 813-815, December 2006.

[12] I. Gradshteyn and I. Ryzhik, Table of Integrals, Series, and Products., 7th ed. ELSEVIER, 2007.

[13] G. P. Efthymoglou, "On the performance analysis of digital receivers in generalized-k fading channels," to be pulished by..., 2010.

[14] L.-L. Yang and H.-H. Chen, "Error probability of digital communications using relay diversity over nakagami-m fading channels," Wireless Communications, IEEE Transactions on, vol. 7, no. 5, pp. 1806-1811, May 2008.

[15] G. L. Stuber, Principles of Mobile Communication. Norwell, MA: Kluwer, 2000.

[16] S. Rice, "Statistical properties of a sine wave plus random noise," Bell Syst. Tech. J., vol. 27, no. 1, pp. 109-157, Jan. 1948. 